\title{
Magnetic Field Models From Energetic Particle Data at Neptune
}

\author{
R. S. SELESNICK \\ Califomia Institute of Technology, Pasadena
}

\begin{abstract}
The locations of features in the Voyager 2 energetic particle data from Neptune are combined with uncertainties in the multipole expansion of the planetary magnetic field to derive new magnetic field models that are consistent both with various interpretations of the particle features and with the magnetic field data. While assumptions as to the origin of the features must be made, they do not provide sufficient constraints to obtain significant new information on any of the unknown multipole coefficients. However, the magnetic $L$ shell positions of the particle features, which are interpreted primarily as absorption signatures of Neptune's satellites, can, in general, be brought into agreement with expected values.
\end{abstract}

\section{INTRODUCTION}

The close encounter of the Voyager 2 spacecraft with Neptune revealed a complex planetary magnetic field [Ness et al., 1989]. To fully represent the data taken by the Voyager 2 magnetometer, an eighth-order multipole expansion was required. However, because of the limitations of the spacecraft trajectory, only a few of the low-order multipole coefficients were able to be accurately resolved from the uncertainties in the remaining coefficients. The best model available from the magnetometer data, the $\mathrm{O}_{8}$ model, contained terms of up to octupole order [Connerney et al., 1991].

The energetic particle experiments on Voyager 2 found a complex radiation environment that was also difficult to interpret in view of the spatially limited data sets [Krimigis et al., 1989; Stone et al., 1989]. In particular, the interpretation of several particle absorption signatures of Neptune's inner satellites was complicated by their relative proximity and the lack of an accurate magnetic field model near the planet. Attempts have been made to understand the origin of the various signatures based on the $\mathrm{O}_{8}$ model [Selesnick and Stone, 1991, 1992; Mauk et al., 1991] with limited success.

An overview of the energetic ( $~(1 \mathrm{MeV})$ electron data taken by the Voyager 2 cosmic ray (CRS) experiment is shown in the top panel of Figure 1. Various features in the data are labeled according to the scheme of Stone et al. [1989], where a detailed discussion of their probable origins can be found. The lower panel of Figure 1 shows the $L$ shell parameter of Voyager 2 based on the $\mathrm{O}_{8}$ model, which, along with the orbital $L$ shells of Neptune's satellites and rings, is the principal tool in an initial interpretation of the particle features. For example, the local electron counting rate minimum, labeled $B$ in the figure, appears to be the result of a local maximum in the spacecraft $L$ that occurred $\sim 11 \mathrm{~min}$ later. However, based on spectral information, Krimigis et al. [1990] suggest that the feature is similar in appearance to signatures of satellite absorption. The local counting rate minimum $I$, also shown in the inset, is almost certainly associated with absorption by the satellite $1989 \mathrm{~N} 1$, but its location does not agree exactly with that predicted by the $\mathrm{O}_{8}$ model on the basis of the satellite minimum orbital $L$ shell locations. The signatures $F$ and $G$ contain, at higher time resolution, many distinct local minima that are probably the result of absorption by the several satellites and rings that orbit in this region [Stone et al., 1989; Selesnick and Stone, 1992]. However,

Copyright 1992 by the American Geophysical Union.

Paper number 92JA00922.

0148-0227/92/92JA-00922\$02.00 an unambiguous identification of the origins of the various signatures has not been achieved.

The goal of this study is twofold. First, it is to understand which, if any, of the possible interpretations of the particle signatures can be consistent with the magnetic field data on the basis of the uncertainties in the $O_{8}$ model. Second, it is to determine whether a given interpretation of the particle data can also provide significant new constraints on any of the magnetic field model parameters. The method of a priori covariances is applied to combine the information from the $\mathrm{O}_{8}$ model and the particle data. Details of the $\mathrm{O}_{8}$ model and the analysis procedure are described in section 2 , followed by discussions of the results and their implications in sections 3 and 4.

\section{METHOD}

\section{The $\mathrm{O}_{8}$ Model}

The $\mathrm{O}_{8}$ magnetic field model was obtained as a partial solution to an eighth-order multipole expansion of Neptune's internal magnetic field plus a uniform external magnetic field, using the singular value decomposition (SVD) method [Connerney et al., 1991]. The model parameters were the 80 multipole coefficients of the internal field plus the three components of the external field. The SVD provides a complete set of eigenvectors in parameter space along which the projections of the parameter vector, the generalized parameters, are independently determined by the magnetic field data. The uncertainties in the generalized parameters are determined by the corresponding eigenvalues. Of the 83 generalized parameters in the model, the 44 most well constrained were included in the final solution. This led to only a few of the multipole coefficients being resolved independently of the remaining 39 generalized parameters, but was necessary in order to prevent large uncertainties in all of the coefficients. However, it is therefore possible to add to the model any reasonable combination of the remaining 39 generalized parameters without significantly degrading the fit to the magnetic field data (this statement will be made quantitative below). A model containing all 83 multipole coefficients derived from the 44-eigenvector partial solution was called the $\mathrm{I}_{8} \mathrm{E}_{1}$ model. However, the $\mathrm{O}_{8}$ model recommended for use by Connerney et al. [1991] contains only the 15 multipole coefficients of third (octupole) order and lower, most, but not all, of which are well resolved. We shall use the $\mathrm{O}_{8}$ model for comparison of our results with those available from the magnetic field data alone, but the full $\mathrm{I}_{8} \mathrm{E}_{1}$ model as a starting point for our calculations.

The covariance matrix of the model parameters (the multipole coefficients) is 


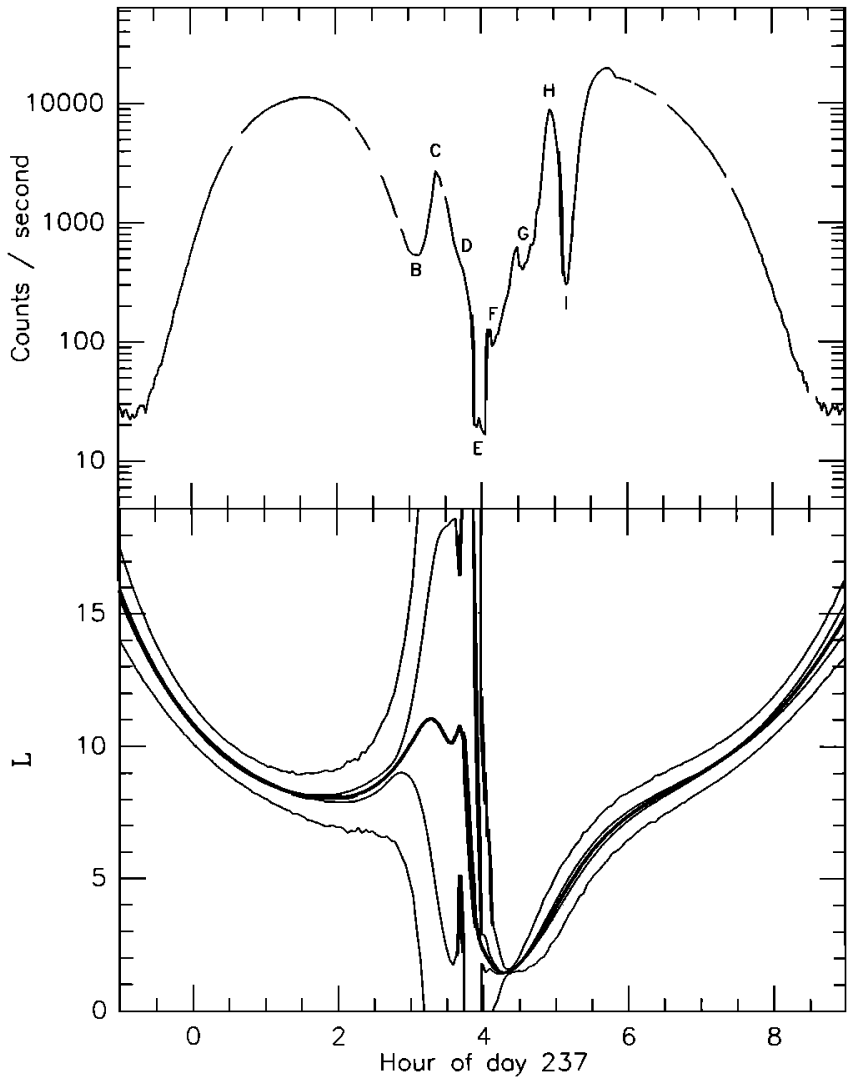

Fig. 1. (Top) Counting rate versus time of $\geq 1-\mathrm{MeV}$ electrons in Neptune's magnetosphere. The letters label features referred to in the text. (Bottom) The Voyager 2 magnetic $L$ shell versus time in the $\mathrm{O}_{8}$ model (heavy curve). The inner and outer error bands (light curves) are based on eigenvectors 57-68 and 69-76, respectively, of the $I_{8} E_{1}$ model.

$$
C_{p p}=V \Lambda^{-2} V^{T}
$$

where $V$ is a matrix containing as columns the eigenvectors corresponding to the generalized parameters that were included in the solution. The diagonal matrix, $\Lambda$, contains the corresponding eigenvalues, and $\Lambda^{-2}$ is the covariance matrix of the generalized parameters. The matrix $C_{p p}$ provides the uncertainties in the model parameters assuming that the 39 generalized parameters corresponding to the eigenvectors not in the solution are all zero. The assumption applies only to the parameters in the model that are unresolved, the others being nearly independent of the 39 generalized parameters. In order to estimate the uncertainties due to the generalized parameters not in the solution it is necessary only to replace $V$ and $\Lambda$ in (1) with matrices made up of the eigenvectors and eigenvalues corresponding to those generalized parameters. With regard to the interpretation of the particle data we calculate the uncertainty (standard deviation), $\sigma_{L}$, in the $L$ shell parameter of the spacecraft, which is given by

$$
\sigma_{L}^{2}=\frac{\partial L^{T}}{\partial p} C_{p p} \frac{\partial L}{\partial p}
$$

where the partial derivatives represent parameter space gradients in the form of column vectors.

Values of $\sigma_{L}$ from (2), versus time, are included as the error bands on the $L$ shell curve in Figure 1. The matrix $C_{p p}$ was calculated from (1) using in $V$ only the eigenvectors 57 to 68 for the inner band and 69 to 76 for the outer band (as labeled in the appendix to Connerney et al. [1991]). The $L$ shell uncertainties in Figure 1 represent $1 \sigma$, or $68 \%$, confidence limits on the linear combination of parameters that produces the maximum local change in $L$ available from the eigenvectors included in the calculation, all other linear combinations of parameters remaining at their maximum likelihood values according to the magnetic field data. When many linear combinations of parameters are allowed to simultaneously vary from their maximum likelihood values, as will be the case in modeling the particle signature locations, the maximum change in $L$ available from a given set of eigenvectors, while remaining in the $68 \%$ confidence region, will be substantially larger. However, the values of $\sigma_{L}$ at each time in Figure 1 are independent. No single model can produce all of the changes and still be within the $68 \%$ confidence region. Also, the large uncertainties in $L$ near hours 3 to 4 are not accurate because the linear approximation under which (2) holds will not be valid there. The two sets of eigenvectors used to calculate the error bands in Figure 1 were chosen because they illustrate the relative uncertainties in $L$ shells at different times, and the increase in the uncertainty as eigenvectors with smaller corresponding eigenvalues are included. Uncertainties resulting from inclusion of all of the eigenvectors are not shown because they become so large that the linear approximation (2) is certainly invalid.

An additional reason for calculating $\sigma_{L}$ from different sets of eigenvectors instead of from the complete set is that, as will become apparent below, including those with the smallest eigenvalues leads to unphysically large high-order magnetic moments. For a spherical harmonic expansion in terms of the Schmidt normalized coefficients $g_{n}^{m}$ and $h_{n}^{m}$, the harmonic spectrum for the $I_{8} E_{1}$ model, defined as a function of the order $n$ by

$$
L_{n}=(n+1) \sum_{m=1}^{n}\left[\left(g_{n}^{m}\right)^{2}+\left(h_{n}^{m}\right)^{2}\right]
$$

is relatively constant, or flat, suggesting that the boundary of the dynamo region is near the surface of the planet [Connermey et al., 1991]. It is reasonable to expect that this property should be preserved by new magnetic field models. The uncertainties in the $\mathrm{I}_{8} \mathrm{E}_{1}$ model do not rule out steeply increasing harmonic spectra, so a flat spectrum must be imposed as an additional constraint. This will be done by restricting the changes in the multipole coefficients to linear combinations of subsets of the eigenvectors corresponding to unconstrained generalized parameters.

\section{Including the Particle Data}

The values of $\sigma_{L}$ in Figure 1 show that there is the possibility of modifying the $\mathrm{O}_{8}$ model in order to be consistent with the various interpretations of the particle data. These interpretations provide tentative constraints on the magnetic $L$ shell parameters of the spacecraft and satellites, which are functions of the model parameters. The goal is to find a set of parameters that predicts the correct $L$ shell behavior according to a given interpretation. This will be achieved by using the requirements of a given interpretation of the particle signatures as data for a new fit of the magnetic field model parameters. A data vector, $d$, is defined to contain the expected $L$ shell behavior. For example, one element of $\mathbf{d}$ may be the difference between the spacecraft $L$ and satellite minimum $L$ at the time of an observed satellite absorption signature. The a priori data vector, $\mathbf{d}_{0}$, contains the measured data values (or, in our case, those assumed for a given interpretation of the particle data), and $C_{d_{d^{d}}{ }_{0}}$ is their covariance matrix. In the previous example, the element of $d_{0}$ corresponding to the difference between the spacecraft $L$ and satellite minimum $L$ 
would contain zero. The parameter vector, $p$, which contains as elements the multipole coefficients of the magnetic field model, leads to a prediction of the data vector, $d$, that can be compared with $\mathbf{d}_{0}$,

$$
\mathbf{d}=\mathbf{g}(\mathbf{p})
$$

where $\mathbf{g}$ is, in general, a nonlinear vector function of $\mathbf{p}$. The problem is to combine (4) with the a priori information available from the $I_{8} E_{1}$ model and solve for a new set of model parameters, p. An iterative solution, based on the least squares criterion, is [Tarantola and Valette, 1982]

$$
\begin{aligned}
\mathbf{p}_{k+1}=\mathrm{p}_{0}+C_{p_{0} p_{0}} G_{k}{ }^{T}\left(C_{d_{0} d_{0}}+G_{k} C_{p_{0^{p}}{ }_{0}} G_{k}^{T}\right)^{-1} \\
\cdot\left[\mathbf{d}_{0}-\mathbf{g}\left(\mathrm{p}_{k}\right)+G_{k}\left(\mathrm{p}_{k}-\mathrm{p}_{0}\right)\right]
\end{aligned}
$$

where $\mathrm{p}_{k}$ is the solution at the $k$ th iteration, and the matrix

$$
G_{k}=\frac{\partial \mathrm{g}\left(\mathbf{p}_{k}\right)}{\partial \mathrm{p}_{k}}
$$

The $\mathrm{I}_{8} \mathrm{E}_{1}$ model parameters and the covariance matrix $C_{p p}$ from (1) become the a priori parameters, $p_{0}$, and covariance matrix, $C_{p_{0}{ }_{0}}$ of the solution (5).

The solution (5) is in a form that is particularly useful in problems that are underdetermined, that is, having more unknowns than data points. The existence of a unique solution is guaranteed by the use of the a priori information, so that if the data are not able to significantly constrain a certain component of the model, then that component remains near its a priori value.

Since the $I_{8} E_{1}$ model provides strong constraints on many of its generalized parameters, it is not necessary to include all of these in the new model, as they will remain largely unchanged. It is convenient to relate the solution (5) to the eigenvectors corresponding to the generalized parameters of the $I_{8} E_{1}$ model that will be included in the new model. We define the parameter increment vector, generalized parameter increment vector, and generalized $G_{k}$ matrix by

$$
\begin{aligned}
& \varepsilon_{k}=\mathrm{P}_{k}-\mathrm{P}_{0} \\
& \varepsilon_{k}^{*}=V^{T} \varepsilon_{k} \\
& G_{k}^{*}=G_{k} V
\end{aligned}
$$

respectively, where $V$ contains only the eigenvectors to be included in the new model. The solution (5) can now be rewritten using (1) as

$$
\begin{aligned}
\varepsilon_{k+1}^{*}=\Lambda^{-2} G_{k} * T & \left(C_{d_{0} d_{0}}+G_{k} * \Lambda^{-2} G_{k} * T\right)^{-1} \\
\cdot & {\left[\mathrm{d}_{0}-\mathbf{g}\left(\mathrm{p}_{k}\right)+G_{k} * \varepsilon_{k} *\right] }
\end{aligned}
$$

Note that $G_{k}{ }^{*}=\partial g / \partial \varepsilon_{k} *$ in (8) can be computed more efficiently than $G_{k}$ in (5) provided that not all of the generalized parameters are included in the solution. The parameters of the new model can be obtained from (7) and the covariance matrix, in the linear approximation, from [Tarantola and Valette, 1982]

$$
\begin{gathered}
C_{p p}=V C_{\varepsilon_{k} * \varepsilon_{k}} \cdot V^{T} \\
C_{\varepsilon_{k} * \varepsilon_{k} *}=\Lambda^{-2}-\Lambda^{-2} G_{k}{ }^{* T}\left(C_{d_{d_{0}} d_{0}}\right. \\
\left.+G_{k} * \Lambda^{-2} G_{k}{ }^{* T}\right)^{-1} G_{k} * \Lambda^{-2}
\end{gathered}
$$

The multipole magnetic field model typically contains many more parameters than there are data points from the particle signatures. Therefore, the model is underdetermined and is constrained primarily by the a priori information. If the data are exact $\left(C_{d_{o^{d}}}=0\right)$ then, after premultiplying by $G_{k}{ }^{*}$, it is easy to see that in the limit $k \rightarrow \infty$ the solution (8) satisfies exactly $g\left(p_{k}\right)=d_{0}$. The solution was derived by minimizing the sum, $\chi^{2}+\delta x^{2}$, of the usual $\chi^{2}$ function and the change in the $\chi^{2}$ function of the 6 t to the magnetic field data that would result from the new model parameters,

$$
\begin{gathered}
\chi^{2}=\left(\mathbf{d}-\mathrm{d}_{0}\right)^{T} C_{d_{0} d_{0}}{ }^{-1}\left(\mathrm{~d}-\mathrm{d}_{0}\right) \\
\delta \chi^{2}=\left(\mathrm{p}-\mathrm{p}_{0}\right)^{T} C_{p_{0} p_{0}}^{-1}\left(\mathrm{p}-\mathrm{p}_{0}\right)=\mathrm{e}^{* T} \Lambda^{2} \varepsilon^{*}
\end{gathered}
$$

Thus, to the extent that the magnetic field data and the particle data conflich, the solution is weighted according to the relative sizes of $C_{p_{0} p_{0}}$ and $C_{d_{0} d_{0}}$. By choosing small values of $C_{d_{0} d_{0}}$ we can assure that if the iterative solution (8) converges, the particle data are accurately modeled. The value of $\delta \chi^{2}$ then determines whether the new model is within a parameter region that is still consistent with the magnetic field data. The $\delta \chi^{2}$ has a $\chi^{2}$ distribution with the same number of degrees of freedom as there are generalized parameters in the solution. The $1 \sigma$, or $68 \%$, confidence region of the $\mathrm{I}_{8} \mathrm{E}_{1}$ model is bounded by a contour in parameter space with a constant value of $\delta \chi^{2}$ that is slightly larger than the number of degrees of freedom. For example, with 12 generalized parameters, the $1 \sigma$ confidence limit is $\delta \chi^{2}=13.7$. Any smaller value means that the parameters are within the $68 \%$ confidence region of the $I_{8} E_{1}$ model.

\section{RESULTS}

Four new magnetic field models will be discussed, based on different particle signatures, on different interpretations of the same signatures, or on different a priori constraints. In each case, the models were derived from the iterative solution (8). The uncertainties in the locations of the particle signatures, corresponding to the square roots of the diagonal elements of $C_{d_{0}{ }^{d}{ }^{0}}$, were assumed to be 0.01 units in $L$, based on the similar accuracy of the $L$ shell calculations. This is well within the accuracy at which the particle signatures can be identified. The off-diagonal elements of $C_{d_{0^{d}} \boldsymbol{d}}$ were assumed to be zero. Only a few iterations of the solution were required in order to achieve a value of $\chi^{2}$ approximately consistent with the assumed uncertainties.

\section{Outer Signatures}

We first consider only the outer two local counting rate minima in the CRS electron data, labeled $B$ and $I$ in Figure 1 . The I signature came outbound from Neptune at a radial distance near 4 $R_{N}\left(1 R_{N}=24,765 \mathrm{~km}\right)$ and, according to the $\mathrm{O}_{8}$ model, at $L \approx 4.7$. At high time resolution the deep counting rate minimum contains a secondary minimum just inside the primary one, as shown in Figure 2. The two minima were observed at $-0508: 30$ and 0510:40 SCET (spacecraft event time). Selesnick and Stone [1991] discuss the possibility that they are caused by strong absorption of electrons by the satellite $1989 \mathrm{~N} 1$ at each of its two orbital minimum $L$ shells. We will assume this to be true. Two simulated data points for the model fit ( $d$ in (4)) are therefore the differences between the spacecraft $L$ at the inner (or outer) counting rate minimum and the corresponding inner (or outer) 


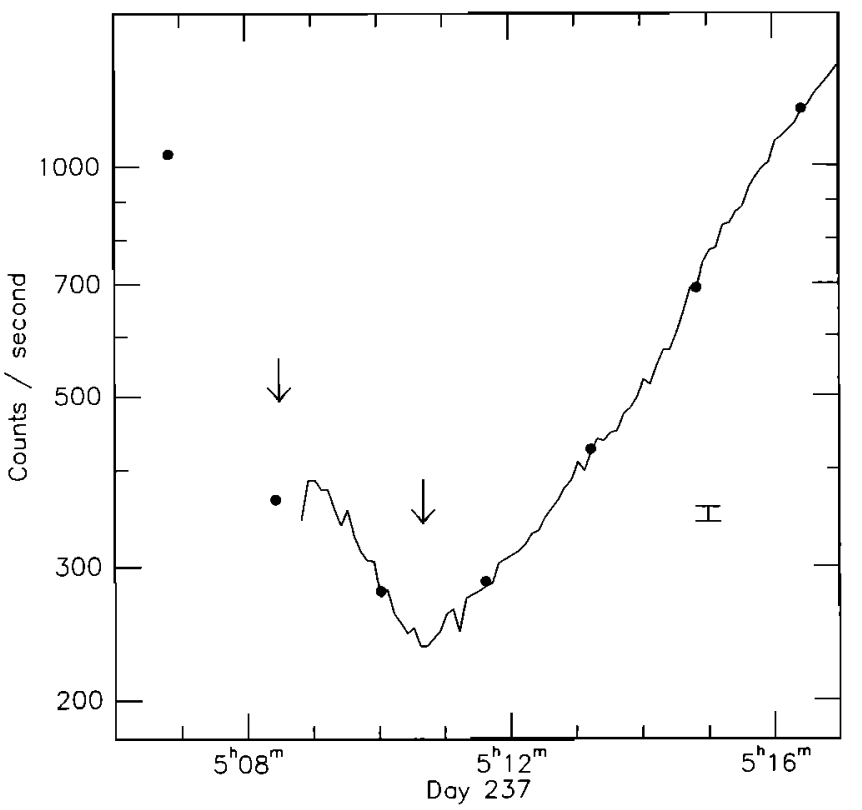

Fig. 2. Close-up of the $\geq 1-\mathrm{MeV}$ electron counting rates near the I signature in Figure 1. The low time resolution (96 s) data points are the same as those in Figure 1, and additional high time resolution $(6 \mathrm{~s})$ data are shown at the times that they are available. The arrows indicate the times of the satellite absorption signatures discussed in the text. A statistical error bar is shown to the right.

minimum orbital $L$ of the satellite. The data values $\left(d_{0}\right)$ are zero. In this and all models we assume for simplicity that the satellite orbits have no eccentricity or inclination so that the two minimum orbital $L$ shells are the same for all orbits. This assumption introduces errors in the signature locations that are generally small compared with the discrepancies between the observed and model locations.

The $B$ signature came inbound to Neptune at a radial distance near $3 R_{N}$ and at -0306 SCET but, according to the $\mathrm{O}_{8}$ model, at a higher magnetic latitude than the I signature with $L=11$. A local maximum in the spacecraft $L$ at the time of the signature could have produced the observed counting rate minimum if it occurred in a region of radially decreasing electron flux, as appears to be the case from the outbound data. The $\mathrm{O}_{8}$ model contains such a local maximum $L$ shell near the required time (Figure 1). A third simulated data point for the first model fit is then the time derivative of the spacecraft $L$ shell evaluated numerically at the time of the signature. The data value is again zero.

Note that because the magnetic latitude of the spacecraft varies with time and the electron intensity may be a strong function of latitude, the locations of the observed signatures can be displaced from the intensity minima or spacecraft $L$ shell turning points. Such displacements are typically small for reasonable electron pitch angle distributions, and we ignore them in this work.

In addition to the three data points described above, the first model fit (model 1 ) includes in $V$ the eigenvectors 69 to 83 , corresponding to the 15 least constrained generalized parameters of the $\mathbf{I}_{8} \mathrm{E}_{1}$ model. The model 1 results are listed in the first column of Table 1 . Shown are the $\chi^{2}$ and $\delta \chi^{2}$ values from (10) and the 15 dipole, quadrupole, and octupole parameter increments, $\varepsilon_{t}$, from the final iteration of the fit. The small value of $\delta \chi^{2}$ shows that model 1 is well within the region of parameter space that is consistent with the $\mathrm{I}_{8} \mathrm{E}_{1}$ model based on the $\mathrm{I}_{8} \mathrm{E}_{1}$ parameter uncertainties.
TABLE 1. For Models 1 to 4 the Model Parameter Increments From the $\mathrm{O}_{8} \mathrm{Model}$ and, for $\mathrm{O}_{8}$, the Model Parameter Values

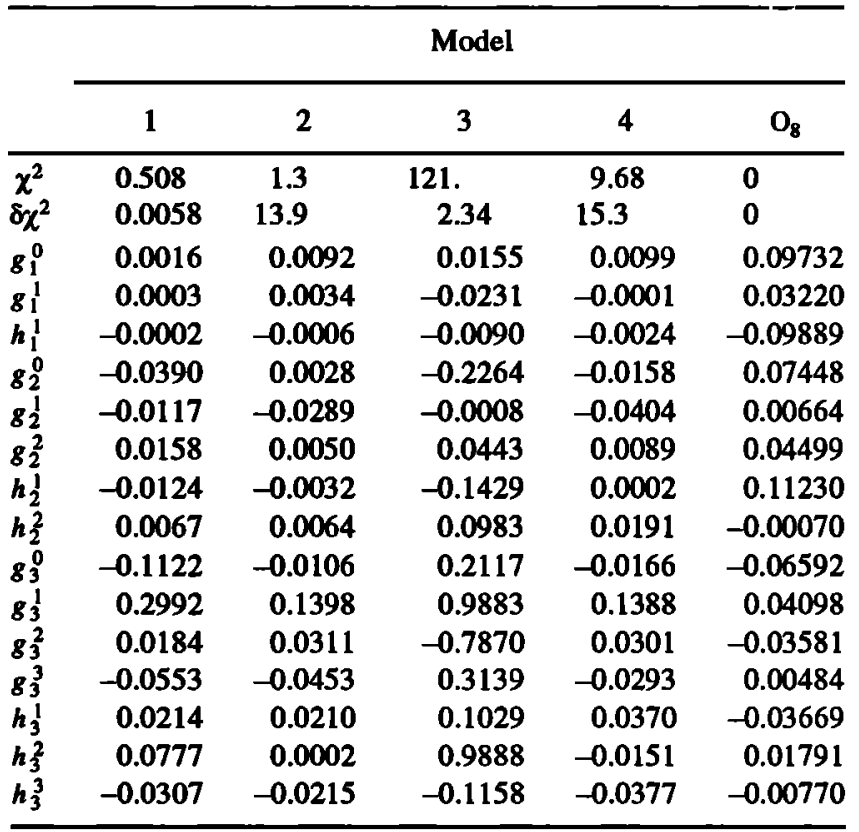

Model 1 achieved an accurate fit to the particle signatures and leads to a simulated magnetic field along the Voyager 2 trajectory that fits the magnetometer data to an accuracy that is virtually indistinguishable from that of the $\mathrm{I}_{8} \mathrm{E}_{1}$ model. However, the parameter values differ greatly from those of the $\mathrm{I}_{8} \mathrm{E}_{1}$ model. This is illustrated in Figure 3, which shows the harmonic spectra, $L_{n}$ versus $n$, of the various models. The $I_{8} E_{1}$ spectrum is relatively flat, while the model 1 spectrum increases rapidly with $\boldsymbol{n}$.

In order to restrict the parameter differences from the $\mathrm{I}_{8} \mathrm{E}_{1}$ model, model 2 uses the same data as model 1 but changes the a priori information by including only eigenvectors 45 to 56 . These correspond to the 12 most constrained of the 39 generalized

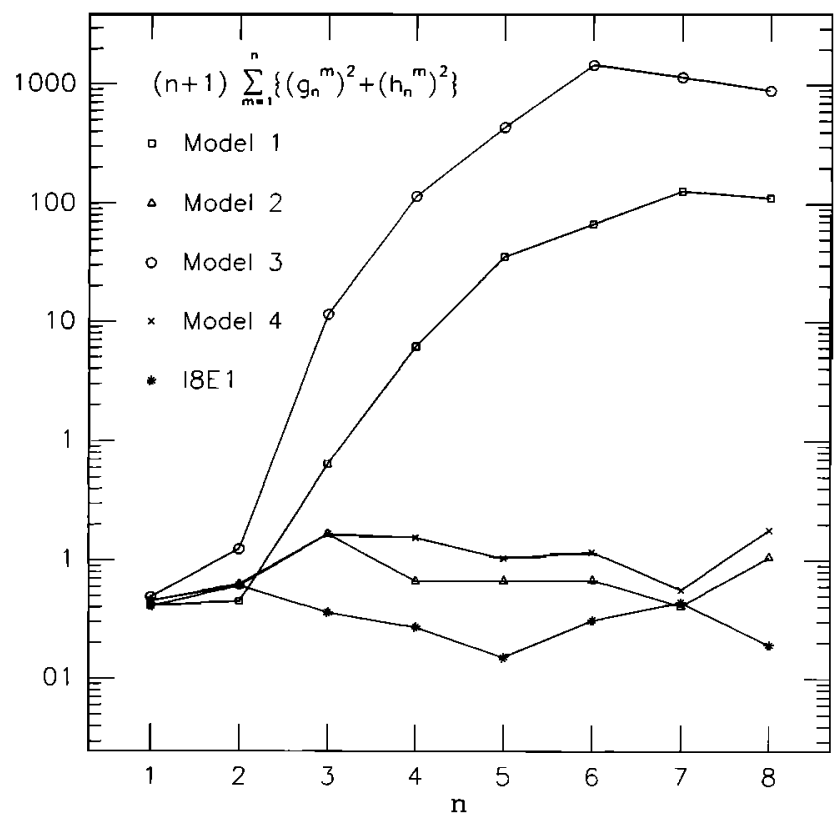

Fig. 3. Harmonic spectra of the various magnetic field models discussed in the text. 


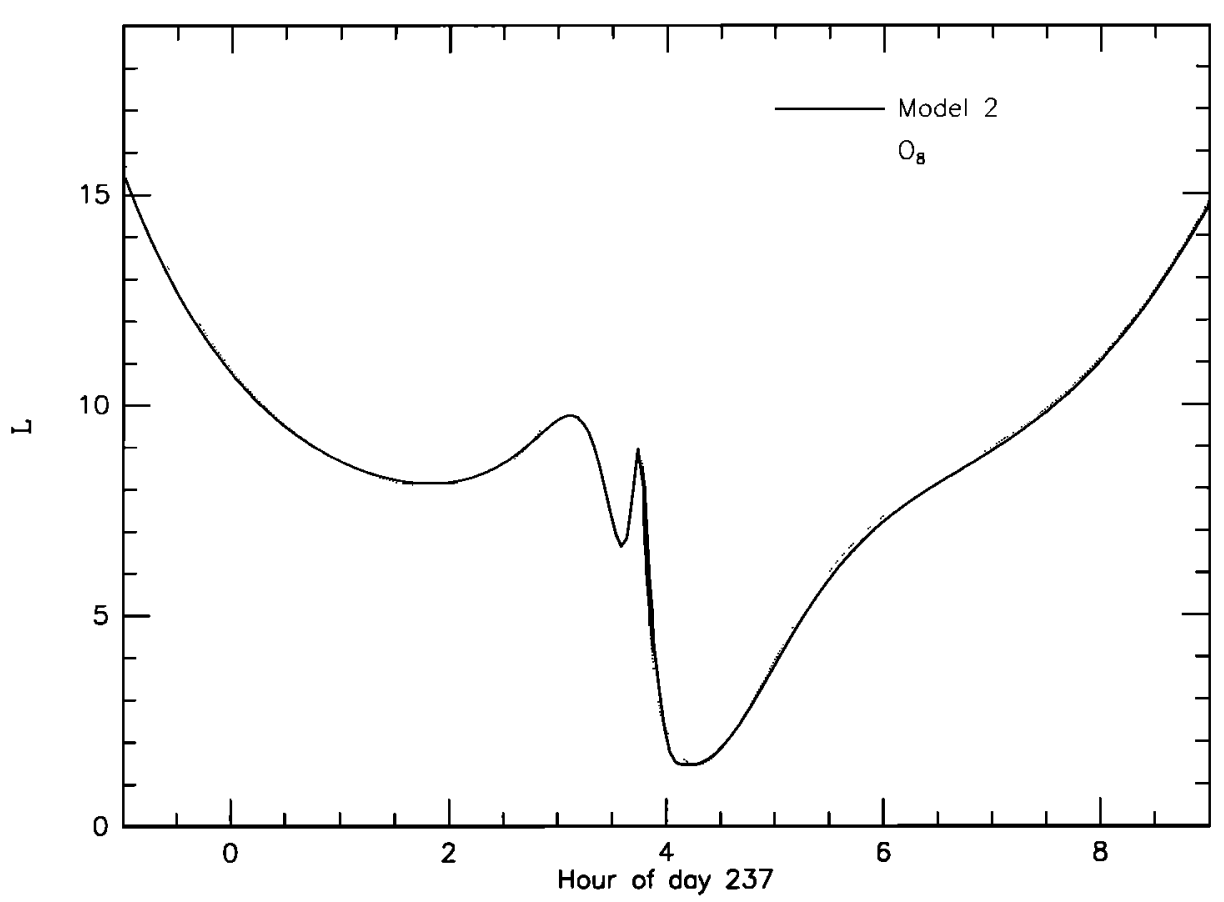

Fig. 4. Mode] Voyager 2 magnetic $L$ shells versus time. Model 2 is based on signatures B and I in Figure 1.

parameters that were not included in the $\mathrm{I}_{8} \mathrm{E}_{1}$ model. The results in Table 1 show that a model substantially different from model 1 is obtained. The $\delta \chi^{2}$, while larger than that of model 1 , is at the $69 \%$ confidence boundary, which means that there is a $69 \%$ probability, according to the $\mathbf{I}_{\mathbf{8}} \mathrm{E}_{\mathbf{1}}$ model, of the true parameters being within this boundary. The model 2 harmonic spectrum in Figure 3, like that of the $\mathrm{I}_{8} \mathrm{E}_{1}$ model, is relatively flat. These results show that the assumed interpretations of the outer particle signatures can be consistent with the magnetic field data and the assumption of a flat harmonic spectrum.

The spacecraft $L$ versus time cómputed from all 80 model 2 parameters is shown in Figure 4, along with the $\mathrm{O}_{8}$ values for comparison. The $\sim 11-\mathrm{min}$ shift in the location of the $L$ shell local maximum to agree with that of the B signature is apparent. The fit to the two data points in the I signature was achieved by decreasing the separation of the two $1989 \mathrm{~N} 1$ minimum orbital $L$ values from $\sim 0.34$ in the $\mathrm{O}_{8}$ model to $\sim 0.15$ in model 2 .

The 12 generalized parameter increments, $\varepsilon^{*}$, are listed in Table 2, from which all of the model 2 parameters could be constructed. Also listed are the corresponding uncertainties from the square roots of the diagonal elements of $C_{\mathrm{e}^{*} \mathrm{e}^{*}}$ given by $(8 b)$, and the a priori uncertainties, $\sigma_{0}$, which are just the inverse eigenvalues. Although some are lower, the uncertainties are generally close to their a priori values. In addition, many other satisfactory models could be found by including more eigenvectors in the fit, as long as the contributions from those with small eigenvalues are restricted to prevent large high-order harmonics. This could be achieved by, for example, artificially increasing the small eigenvalues in the solution (8). Thus the model 2 solution is both poorly constrained and nonunique.

An alternative interpretation of the B signature is that, like the I signature, it is due to absorption by $1989 \mathrm{~N} 1$. This would require a large departure of the spacecraft $L$ shells from those predicted by the $\mathrm{O}_{8}$ model. $A$ data point concerning signature $B$ corresponding to the alternative interpretation is the difference between the spacecraft $L$ and the outer 1989N1 minimum orbital
$L$ (high time resolution CRS data are not available in signature B), and its value is zero. Several attempts were made to fit the new data point along with the same data points concerning the I signature as were used in models 1 and 2 . In no case were both a good fit and a flat harmonic spectrum achieved. One example is model 3, which included eigenvectors 35 to 76 . A reasonable fit to the data points was obtained, but at the expense of large highorder harmonics. The interpretation of signature $B$ as an absorption signature of $1989 \mathrm{Nl}$ is therefore unlikely. However, because of the nonlinearity of the $L$ shell calculations, it cannot be ruled out entirely without a systematic search of the parameter space.

\section{Inner Signatures}

The inner signatures ( $F$ and $G$ in Figure 1) are more difficult to interpret because, along with the availability of many potential particle absorbers, their locations are energy dependent [Mauk et

TABLE 2. Generalized Model Parameters and Standard Deviations

\begin{tabular}{ccrrrr}
\hline $\begin{array}{l}\text { Eigen- } \\
\text { vector }\end{array}$ & $\sigma_{0}$ & Model 2 & $\sigma_{2}$ & Model 4 & $\sigma_{4}$ \\
\hline 45 & 0.0067 & 0.0047 & 0.0065 & -0.0017 & 0.0067 \\
46 & 0.0106 & 0.0140 & 0.0089 & -0.0079 & 0.0103 \\
47 & 0.0147 & -0.0069 & 0.0145 & 0.0095 & 0.0143 \\
48 & 0.0202 & -0.0161 & 0.0191 & 0.0270 & 0.0187 \\
49 & 0.0226 & 0.0152 & 0.0222 & 0.0043 & 0.0224 \\
50 & 0.0262 & 0.0511 & 0.0226 & 0.0079 & 0.0254 \\
51 & 0.0334 & 0.0138 & 0.0304 & 0.0085 & 0.0284 \\
52 & 0.0377 & -0.0069 & 0.0375 & 0.0600 & 0.0338 \\
53 & 0.0525 & 0.0053 & 0.0400 & -0.0485 & 0.0480 \\
54 & 0.0592 & 0.0399 & 0.0482 & 0.0041 & 0.0464 \\
55 & 0.0942 & -0.1480 & 0.0709 & -0.0649 & 0.0566 \\
56 & 0.1067 & 0.1983 & 0.0617 & 0.3105 & 0.0527 \\
\hline
\end{tabular}




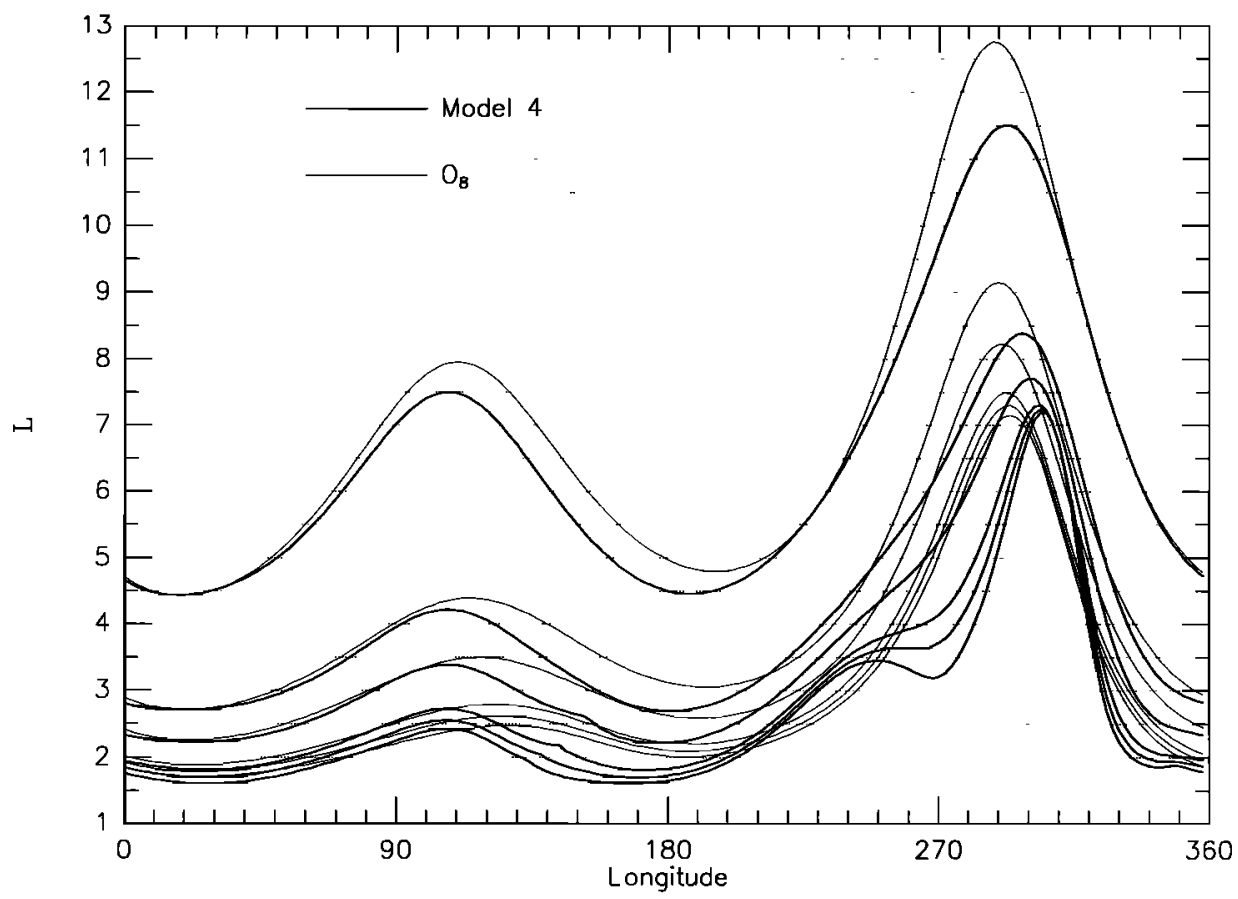

Fig. 5. Model satellite magnetic $L$ shells versus longitude. Model 4 is based on the assumption that two local minima for each satellite are equal.

al., 1991; Selesnick and Stone, 1992]. Detailed modeling of the absorption process by satellites and rings will probably be required to fully understand these signatures. However, the CRS signatures appear to be reasonably well organized by the relative locations of the satellites and rings if it is assumed that they each produce a single absorption signature at $L$ shells that are spaced similarly to their orbital radii [Stone et al., 1989; Selesnick and Stone, 1992]. This assumption would appear to require that the magnetic field model be more symmetric than the $\mathrm{O}_{8}$ model in the sense that the two orbital minimum $L$ shells of each satellite be equal. We therefore adopt as six data points for model 4 the differences of the two orbital minimum $L$ shells of the satellites $1989 \mathrm{~N} 1$ to $1989 \mathrm{~N} 6$. Note that the model 4 assumptions regarding signature I are slightly different than those of models 1, 2 and 3. Again, the data values are all zero and, as in model 2 , the fit includes only the eigenvectors 45 to 56 in order to obtain a flat harmonic spectrum.

The model 4 results are listed in Tables 1 and 2 , and the six satellite $L$ shells versus longitude for both model 4 and the $\mathrm{O}_{8}$ model are shown in Figure 5 , which verifies that the fit has achieved equality in the minimum $L$ shell values of each satellite. The value of $\delta \chi^{2}$ corresponds to a $77 \%$ confidence boundary, so that an interpretation of the signatures based on the model 4 assumptions can be consistent with the magnetic field data. The same conclusions as in model 2 concerning the accuracy and uniqueness of the solution apply here. These results show that in any calculation of the particle signature locations, substantial adjustments can be expected on the basis of uncertainties in the $\mathrm{O}_{8}$ model. However, the somewhat speculative nature of the particular interpretation of the particle signatures used in model 4 should also be kept in mind.

\section{Discussion}

We have seen that it is possible to combine the information available from the magnetic field data with new information based on the locations of the particle signatures to obtain new magnetic field models that are consistent with both data sets. The particle signatures add only a few data points that are insufficient to provide significant new constraints on the many field model parameters, most of which have large uncertainties. The search for new field models is limited primarily by the nonlinear relationship between the model parameters and the locations of the particle signatures. However, when the nonlinearity is not too large, the iterative solution converges rapidly.

In order to obtain physically reasonable high-order magnetic moments, it was necessary to restrict the model solution in the parameter space directions that are largely unconstrained by the magnetic field data alone. The application of this extra a priori information led to magnetic field models that may be closest to the true planetary magnetic field, but again the models are not unique. Additional reliable information of this sort is required to improve the models and may be available from more detailed analyses of the particle absorption mechanisms by Neptune's inner satellites and rings.

Acknowledgments. I thank J. E. P. Connerney for helpful discussions. This work was supported by NASA under contract NAS7-918 and grant NAGW-2402.

The editor thanks J. W. Belcher and D. Hamilton for their assistance in evaluating this paper.

\section{REFERENCES}

Connerney, J. E. P., M. H. Acuna, and N. F. Ness, The magnetic field of Neptune, J. Geophys. Res., 96, 19,023-19,042, 1991.

Krimigis, S. M., T. P. Armstrong, W. I. Axford, C. O. Bostrom, A. F. Cheng, G. Gloeckler, D. C. Hamilton, E. P. Keath, L. J. Lanzerotti, B. H. Mauk, and J. A. Van Allen, Hot plasma and energetic particles in Neptune's magnetosphere, Science, 246, 1483-1489, 1989.

Krimigis, S. M., B. H. Mauk, A. F. Cheng, E. P. Keath, M. Kane, T. P. Armstrong, G. Gloeckler, and L. J. Lanzeroti, Hot plasma parameters in Neptune's magnetosphere, Geophys. Res. Lett., 17, 1685-1688, 1990.

Mauk, B. H., E. P. Keath, M. Kane, S. M. Krimigis, and A. F. Cheng, The hot plasma and radiation environment of Neptune, J. Geophys. Res., 96, 19,061-19,084, 1991. 
Ness, N. F., M. H. Acuna, L. F. Burlaga, J. E. P. Connerney, R. P. Tarantola, A. and B. Valette, Generalized nonlinear inverse problems Lepping, and F. M. Neubauer, Magnetic fields at Neptune, Science, 246, 1473-1478, 1989.

Selesnick, R. S. and E. C. Stone, The electron absorption signature of 1989N1, J. Geophys. Res., 96, 19,137-19,147, 1991.

Selesnick, R. S. and E. C. Stone, Energetic particle signatures of satellites and rings in Neptune's magnetosphere, Adv. Space Res., in press, 1992.

Stone, E. C., A. C. Cummings, M. D. Looper, R. S. Selesnick, N. Lal, F. B. McDonald, J. H. Trainor, and D. L. Chenette, Energetic charged particles in the magnetosphere of Neptune, Science, 246, 1489-1494, 1989.

(Received October 21, 1991;

revised March 20, 1992;

accepted March 27, 1992.)

R. S. Selesnick, 220-47 California Institute of Technology, Pasadena, CA 91125. 